\title{
Chest Pain and Troponin leak in a Healthy 38-YEAR-OLD FEMALE
}

\author{
Gunjan Shah, MD
}

A 38-year-old female with a medical history significant only for a cholecystectomy for cholelithiasis presented to an outside hospital with a one day history of sharp, non-radiating midsternal chest pain that began when she bent over to pick something up. She has associated light-headedness and dizziness when she stood up, as well as palpitations, diaphoresis, and a sense of throbbing in her left arm. The symptoms persisted at rest for one hour before the patient arrived at the outside hospital. She was found to be in new-onset atrial fibrillation with a rapid ventricular rate up to 160 beats per minute. A diltiazem infusion was started which resulted in a decrease of the heart rate to 100 beats per minute. Her first troponin was $<0.05 \mathrm{ng} /$ $\mathrm{mL}$, but a subsequent troponin was $0.59 \mathrm{ng} / \mathrm{mL}$, prompting the initiation of a heparin infusion for acute coronary syndrome. At this point, she was transferred to Thomas Jefferson University Hospital for further management. Her chest pain had resolved without any other medication, and she remained chest pain free through the rest of her hospital course. Of note, she admitted to using alcohol, tobacco, and cocaine two days prior to admission. Her only medication is a daily oral contraceptive pill.

Her initial vital signs on transfer were a temperature of 97.9 degrees Fahrenheit, blood pressure of 97/63 mm Hg, heart rate on 112 beats per min, which was irregularly irregular, and respiratory rate of 20 breaths per minute with an oxygen saturation of $98 \%$ on room air. Her physical exam was significant for an enlarged left palatine tonsil, irregular heart rate, and ecchymosis on her neck.

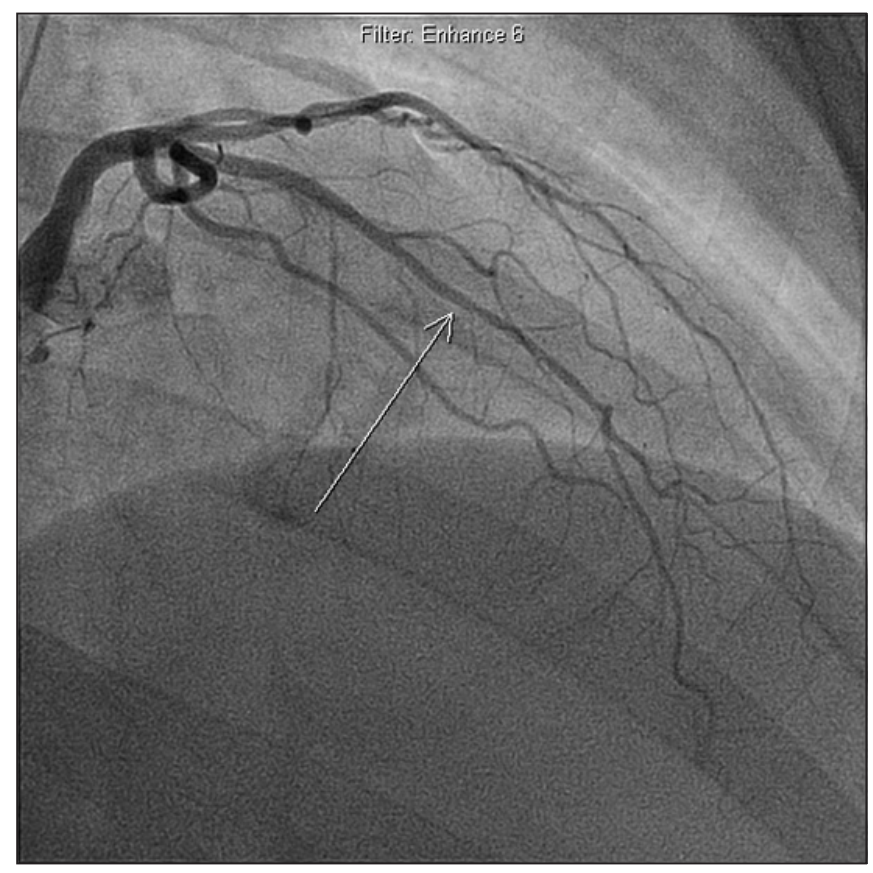

Initial laboratory studies included a white blood cell count of $12,000 / \mathrm{L}$, hemoglobin of $14.1 \mathrm{~g} / \mathrm{dL}$, platelet count of 269,000/L, creatinine of $1.1 \mathrm{mg} / \mathrm{dL}$, AST $48 \mathrm{IU} / \mathrm{L}$, and ALT $41 \mathrm{IU} / \mathrm{L}$. Her maximum troponin was $0.59 \mathrm{ng} / \mathrm{mL}$.

Subsequently, her rhythm converted to sinus rhythm. Her EKG showed sinus tachycardia, but no ST-T changes concerning for myocardial ischemia or infarction.

A cardiac catheterization was done with images as shown below.

Questions: Select the one lettered answer that is BEST in each question.

1. What is the most likely cause of her elevated troponin?
a. Myocardial Infarction
b. Cocaine use
c. Myocardial Bridging
d. Atrial Fibrillation

2. What factor is the most likely determinant of clinical significance?
a. Length of tunneled artery
b. Degree of systolic narrowing
c. Location of artery
d. Width during diastole

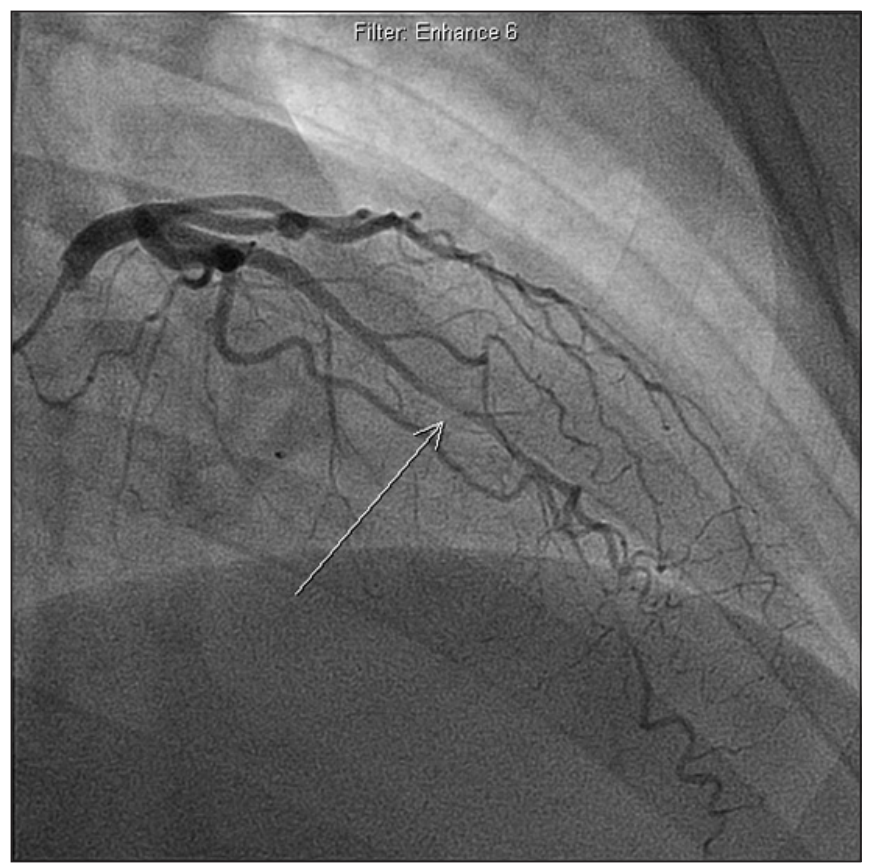

Figure 1. (left) Diastole; (right) Systole 
3. What is the initial treatment of choice?
a. Beta-blockade
b. Coronary stenting
c. Aspirin
d. Surgical myotomy

\section{Discussion}

Myocardial bridging (MB) is a congenital condition in which the normally epicardial coronary artery is tunneled through the myocardium. Its clinical significance remains unclear, but various case reports have associated it with myocardial ischemia, myocardial infarction, arrhythmias, and sudden death. ${ }^{1,5}$ By autopsy, the prevalence ranges between 5.4 and $85 \%$, while by coronary angiography, $0.5-29.4 \% .^{2,4}$ It most commonly affects the mid-portion of the left anterior descending artery (although there are a few case reports where it involves the right coronary artery.). ${ }^{1,4}$ The condition was first described by Reyman in 1737 and is characterized by systolic compression of the artery. ${ }^{3,4}$

One proposed mechanism for myocardial bridging causing myocardial ischemia is its association with coronary vasospasm. ${ }^{2}$ Another theory relies on the amount of blood flow during systole and diastole in the coronary vessels. Stress and exercise can increase the body's sympathetic activity leading to tachycardiainduced ischemia. ${ }^{4}$ Tachycardia shortens the diastolic filling time and increases the importance of systolic filling, which accounts for only $15 \%$ of coronary blood flow. ${ }^{2}$ Furthermore, atherosclerotic plaque typically forms in the artery proximal to the bridge, with relative sparing of the tunneled segment. ${ }^{4}$

As resting EKGs are usually normal, other imaging modalities must be used to investigate the presence and significance of myocardial bridging. Coronary angiography is the gold standard with systolic compression of the tunneled segment portrayed as a "milking effect" and "step down-step up" phenomenon. ${ }^{4}$ Newer studies have been using other invasive imaging techniques such as intravascular ultrasound in which a "half-moon" phenomenon is seen or intracoronary Doppler ultrasound, in which a "fingertip phenomenon" or "spike-and-dome pattern is seen. ${ }^{4}$ Noninvasive imaging is possible with electron beam tomography multi-slice CT, magnetic resonance tomography, or transthoracic Doppler echocardiography, but the sensitivity and specificity preclude these from being the modalities of choice. ${ }^{4}$ Investigations have been conducted using myocardial perfusion imaging, and it has been found that the myocardial ischemia that results from bridging is associated more closely with the degree of systolic narrowing than with the length of tunneled artery or the location of MB. ${ }^{1}$

Therapy for myocardial bridging concentrates on symptom management, with medical therapy being first line. Negative ionotropic and chronotropic agents such as beta-blockers and calcium-channel blockers increase the diastolic perfusion time, thereby decreasing compression of the coronary arteries. Nitrates are often avoided because they angiographically exacerbate myocardial bridging. Coronary stenting of the tunneled vessel and surgical myotomy are reserved for patients who fail medical management. ${ }^{2,4} \mathrm{~W}$ ith these modalities, long-term prognosis in patients with isolated myocardial bridging is generally good. ${ }^{4}$

Answers: $1-C, 2-B, 3-A$

\section{References}

1. Tang Kun, Wang Ling, Shi Rongfang, et al. The role of myocardial perfusion imaging in evaluating patients with myocardial bridging. J Nucl Cardiol. 2010; epub.

2. Vales Lori, Kanei Yumiko, Fox John. Coronary Artery Occlusion and Myocardial Infarction Caused by Vasospasm within a Myocardial Bridge. J Invasive Cardiol. 2010; 22: e67-e69

3. Reyman HC. Disertatio de vasis cordis propriis. Med Diss Univ Göttingen. 7th Sept 1737;1-32.

4. Möhlenkamp S, Hort W, Ge J, et al.Update on myocardial bridging. Circulation. 2002 Nov 12;106(20):2616-22.

5. Sunnassee A, Shaohua Z, Liang R, et al. Unexpected death of a young woman: is myocardial bridgingsignificant?-A case report and review of literature. Forensic Sci Med Pathol. 2010 Aug 10. Epub.

6. Kim PJ, Hur G, Kim SY, et al. Frequency of myocardial bridges and dynamic compression of epicardial coronary arteries: a comparison between computed tomography and invasive coronary angiography. Circulation. 2009;119:1408-1416. 